OPEN ACCESS

Edited by:

Hugo Morais,

University of Lisbon, Portugal

Reviewed by:

Elżbieta Macioszek,

Silesian University of Technology,

Poland

Tiago Pinto,

University of Salamanca, Spain

Yibo Wang,

Northeast Dianli University, China

Teng Li,

State Grid Corporation of China

(SGCC), China

*Correspondence:

Liu Shengli

1072349123@qq.com

Specialty section:

This article was submitted to

Process and Energy Systems

Engineering,

a section of the journa

Frontiers in Energy Research

Received: 02 July 2021

Accepted: 10 September 2021

Published: 14 October 2021

Citation:

Shengli L, Jun W, Longjiang X, Di W, Haiqing $L$, Wenlong $L$, Shaofang $W$ and Wenqi $L$ (2021) Research and

Application of a Clear Diamond-

Shaped Distribution

Network Structure.

Front. Energy Res. 9:735294. doi: 10.3389/fenrg.2021.735294

\section{Research and Application of a Clear Diamond-Shaped Distribution Network Structure}

\author{
Liu Shengli ${ }^{1 *}$, Wu Jun ${ }^{1}$, Xue Longjiang ${ }^{1}$, Wu Di $^{1}$, Lu Haiqing ${ }^{2}$, Li Wenlong ${ }^{3}$, Wang Shaofang ${ }^{3}$ \\ and Li Wenqi ${ }^{3}$
}

${ }^{1}$ State Grid Zhejiang Electric Power Co., Ltd. Pinghu Power Supply Company, Zhejiang Pinghu, China, ${ }^{2}$ State Grid Zhejiang Electric Power Research Institute, Zhejiang Hangzhou, China, ${ }^{3}$ Tianjin Tianda Qiushi New Power Technology Co., Ld., Tianjin, China

Aiming at the problems of low power supply reliability, poor transfer capacity between stations, and low line utilization in the current distribution network, this paper proposes a diamond-shaped distribution network structure with a clear structure. First, we investigated the typical wiring patterns of medium-voltage distribution networks in Tokyo, Japan, Paris, France, and China's developed cities, and summarized experience and shortcomings. Secondly, combining the typical wiring patterns of distribution networks in China and abroad, construct a diamond-shaped distribution network structure, and study its adaptability, safety and flexibility, power supply reliability, and economy. Finally, take the transformation of the wiring mode of a regional distribution network in a certain city as an example, compare the use of the diamond-shaped distribution network structure in this article with the use of cable doublering network wiring, cable "double petal" wiring, and Shanghai diamond-type wiring distribution network grid reconstruction The effect verifies the superiority of the diamond-shaped distribution network structure in this paper.

Keywords: Shanghai diamond wiring, cable "double petal" connection, cable double loop network connection, diamond distribution network, clear structure

\section{INTRODUCTION}

The optimization of the distribution network grid structure is the core content of the distribution network planning and transformation. The wiring mode reflects the basic situation of the grid structure. Therefore, the choice of wiring mode has a significant impact on the reliability, economy, and applicability of the distribution network construction.

Typical problems existing in the urban medium-voltage distribution network in China are: power supply reliability (Lin and Lin, 2020; Li et al., 2021) still has a gap compared with developed countries. Load transfer capacity (Feng et al., 2015; Zhao and Wu, 2016) is insufficient, and the $10 \mathrm{kV}$ line utilization rate (Zhang and Dou, 2018; Xiao and She, 2019) is generally low. The impact of new elements such as distributed energy and microgrid system (Chen and Liu, 2020; Zhou et al., 2021a; Liu et al., 2021; Zhang and Troitzsch, 2021) on the relay protection of the distribution network (Xu, 2017) is not considered. There are more dedicated line users in direct substations, which affects the $10 \mathrm{kV}$ line interval utilization ( $\mathrm{Hu}$ and $\mathrm{Li}, 2017$ ), etc.

At present, experts and scholars have done a lot of research on the wiring mode of the distribution network. The $22 \mathrm{kV}$ cable three-shot connection in Tokyo, Japan has a high line utilization rate, but the load transfer and balance capacity between stations is insufficient (Shang et al., 2015; Liang et al., 
2018). The $20 \mathrm{kV}$ three-ring network connection in Paris, France has high power supply reliability, but the line utilization rate is low, and the economy is poor (Narayanan et al., 2021; Pragathi et al., 2021); developed cities in China use ring network room cable double-ring network connection, which has high power supply reliability. But the line utilization rate is low, and there is no relay protection device, self-healing device, etc., which leads to a wide range of power outages and longer power outages in the event of a fault (Song et al., 2015; Liu et al., 2019). The cable "double petals" connection in Xiong'an New Area has high power supply reliability, $100 \%$ load transfer capacity, and line utilization rate up to $75 \%$. However, the line construction is large, the switching station cost is high, the investment is large, and the operation and maintenance management are more complicated. (Wang and Jiang, 2020). Shanghai diamond type wiring is in the core of the grid structure with the switch station, it uses two-sided power supplies from different substations and construct a doublelayer double-ring network structure. The power supply has high reliability and strong transfer capacity, but the line utilization rate is only $50 \%$. The switch station has a relatively high cost and relatively large investment (Ma et al., 2015)

In order to solve the above-mentioned typical problems in the distribution network structure, this paper proposes a diamondshaped distribution network structure with a clear structure to provide new ideas for urban distribution network planning and transformation.

In order to solve the above-mentioned typical problems of the distribution network structure, this paper proposes a clear structure of the diamond-shaped distribution network structure, and comprehensively analyzes the diamond-shaped mode of this paper from the aspects of adaptability, safety and flexibility, power supply reliability, and economy. This paper provides new ideas for urban distribution network planning and transformation.

\section{RESEARCH STATUS OF MEDIUM VOLTAGE DISTRIBUTION NETWORK IN CHINA AND ABROAD}

\section{Research Status of Foreign Medium Voltage Distribution Network}

1) Tokyo three-shot wiring

The $22 \mathrm{kV}$ cable in the core area of Tokyo adopts a three-radio connection with user dual access. The medium voltage users are all dual power users, and the two power sources come from any two of the three rays. Among them, one is the main power supply and the other is the hot standby power supply. The line load rate is up to $67 \%$.

Under normal operating conditions, it is powered by the main power supply. When the main power supply is out of power or overhauled, the hot standby power supply can be supplied by switching the circuit breaker to achieve the purpose of shortening the power outage time. The wiring diagram is shown in Figure 1.

The main transformer and the supply lines in the three-fire connection station are running in parallel, so that there is no instantaneous power outage when a single line failure occurs, but

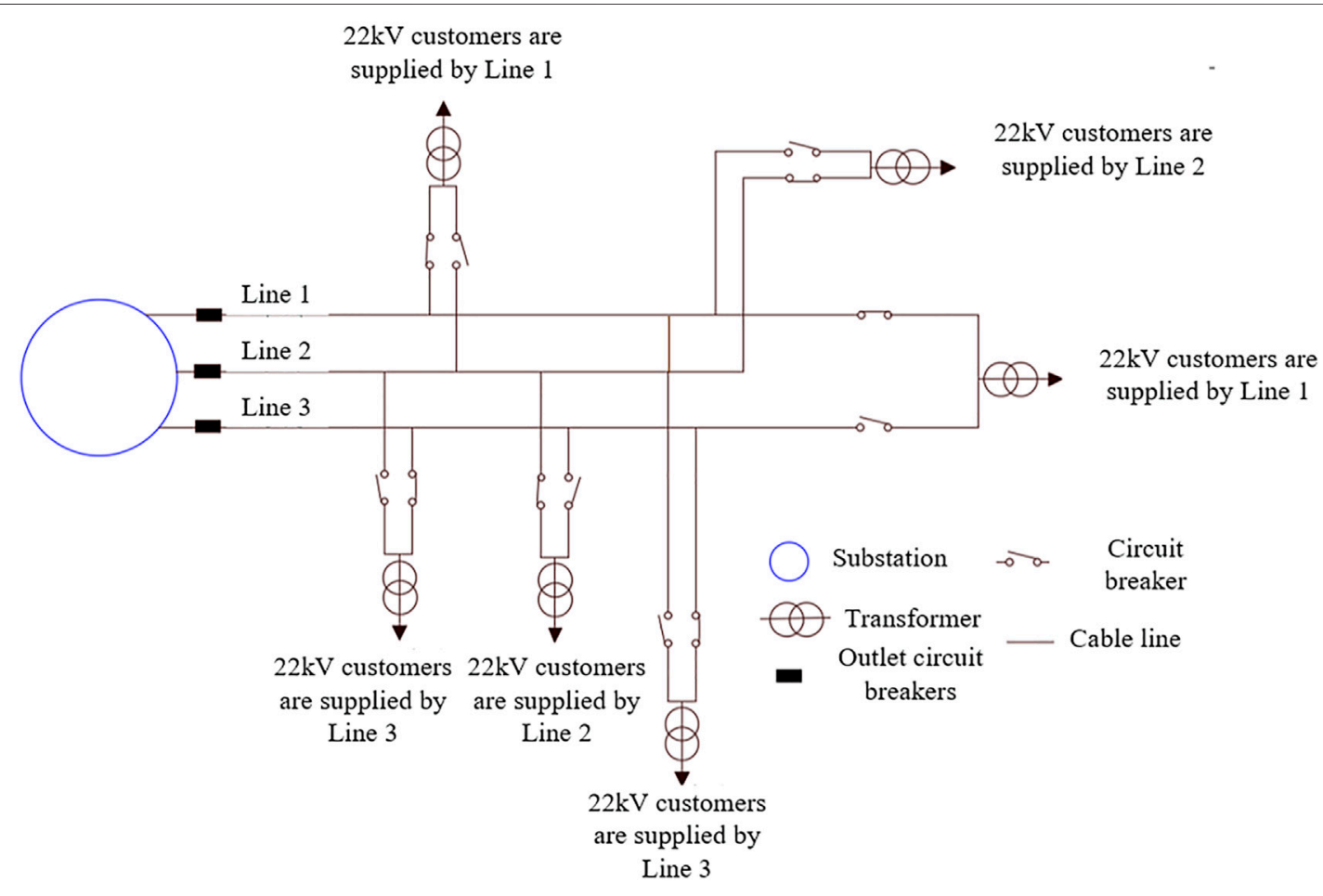

FIGURE 1 | Tokyo $22 \mathrm{kV}$ cable three-shot wiring diagram. 


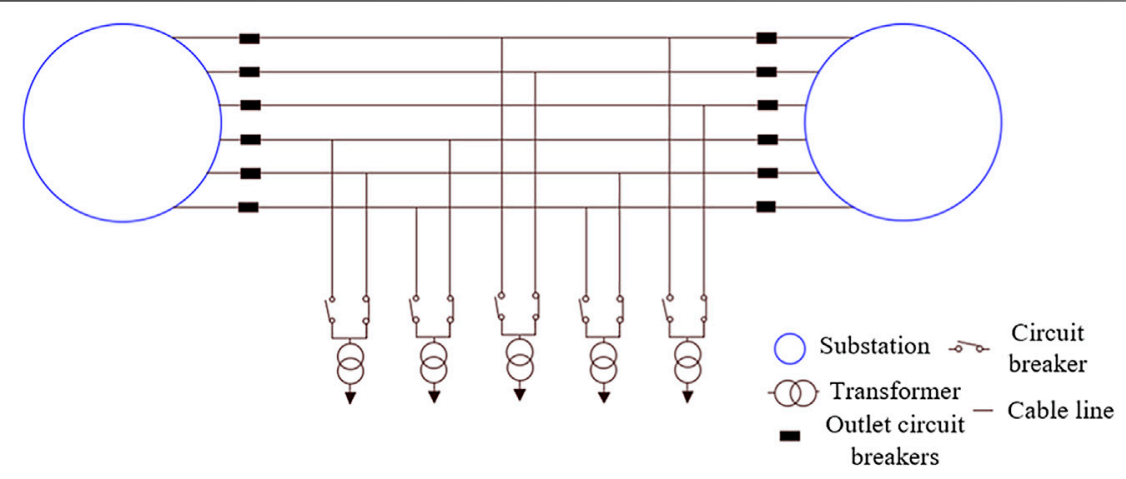

FIGURE 2 | Schematic diagram of 20 kV three-ring network in Paris city.

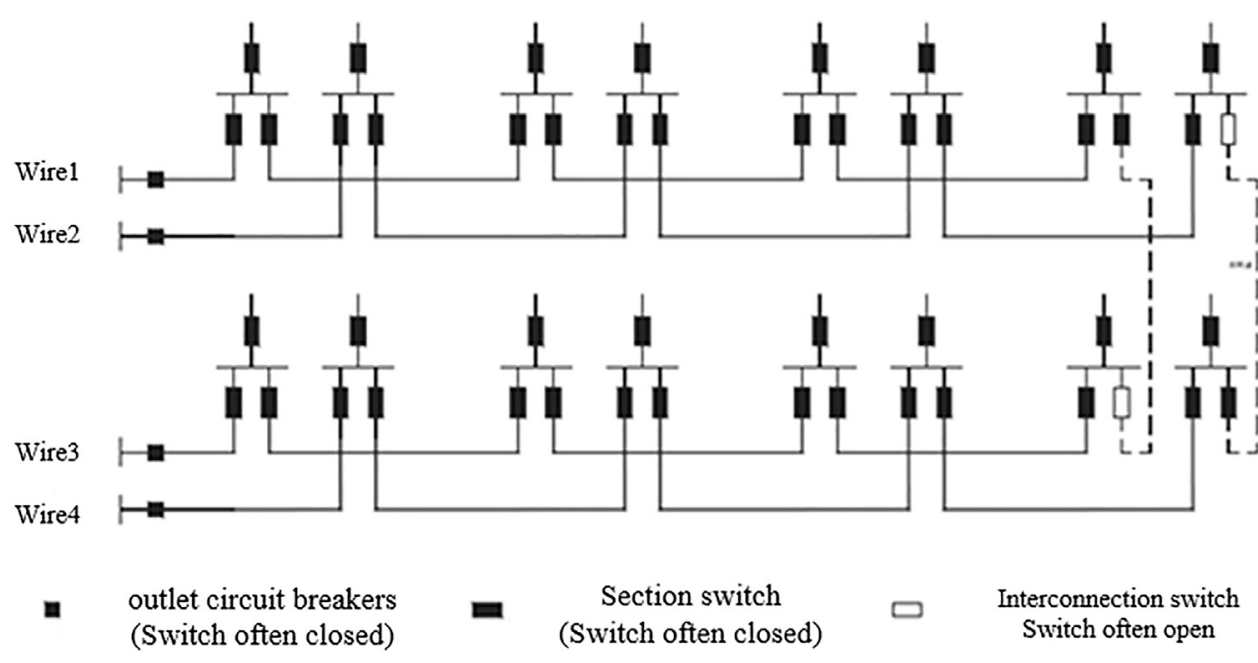

FIGURE 3 | Schematic diagram of cable double ring network connection (ring network room networking).

there is a lack of line connection between the substations, and the load transfer and balance capacity between the stations is insufficient. When the whole station is out of power, all loads will lose power.

2) Wiring of the third ring network in Paris

Most of the $20 \mathrm{kV}$ power distribution network in the urban area of Paris is connected by a cable three-ring network. The wiring diagram is shown in Figure 2. It consists of two sets of cables and three-ray connections, and the power grid operates in an open loop. The user access method is the same as that of Tokyo three-radio wiring.

The section switch and tie switch of the three-ring network have remote control functions. The main and standby power cables of the medium/low voltage distribution room are directly connected to the main cable. When the cable fails, the main power supply load switch of the distribution room is switched off at the substation. After $3 \mathrm{~s}$, the gate will be opened, and the standby load switch will be closed in $5 \mathrm{~s}$, and the power supply of the power distribution room will be restored, which is completed by the automatic device of the equipment.

The reliability of the power supply of the three-ring network in the urban area of Paris is relatively high, but the line utilization rate is low. The maximum utilization efficiency is $33 \%$, and the average utilization efficiency is less than $20 \%$.

\section{Research Status of China's Medium Voltage Distribution Networks}

1) Double ring network connection of cable in ring network room

The core areas with high requirements for China's power supply reliability generally adopt cable double-ring network connection. Cable double-ring network wiring requires lines to come from two substations or different sections of the bus in the same substation. The double ring network connection of the cable in the ring network room is shown in Figure 3. 


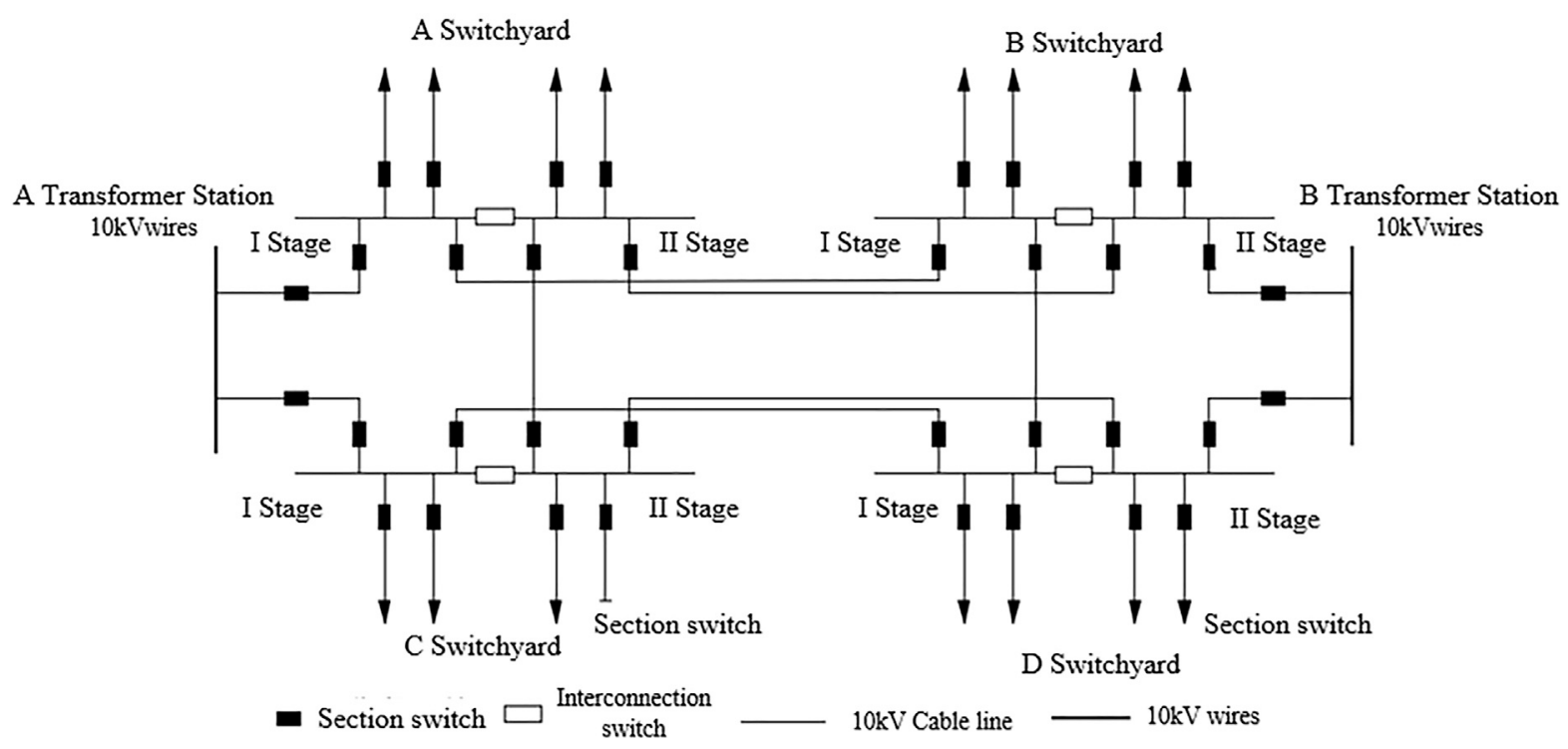

FIGURE 4 | Cable "double petals" wiring diagram.

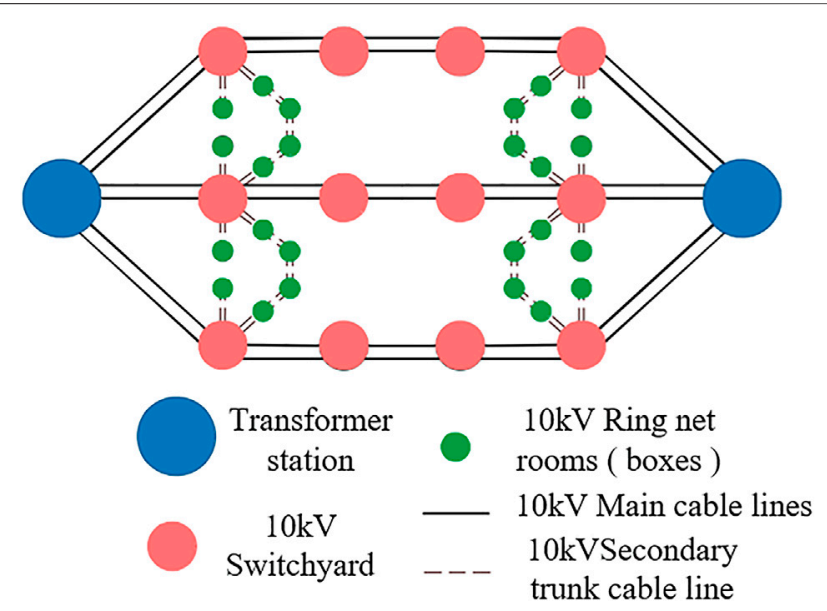

FIGURE 5 | Shanghai diamond wiring diagram.

On the premise of satisfying N-1 (Zhou et al., 2021b), the load rate of the main trunk line during normal operation is up to $50 \%$. This wiring provides users with dual power supply, which can meet the N-1 calibration of $10 \mathrm{kV}$ lines, with high power supply reliability and more flexible operation. Multiple ring network rooms can be connected in series in a double ring network, but the load switch in the ring network room is generally not equipped with a relay protection device, resulting in a wide range of power outages and longer power outages in the event of a fault.

2) Cable "double petals" wiring

The medium-voltage power distribution network frame in Xiong'an New Area uses the "double petals" connection of cables, as shown in Figure 4. The cable "double petals" structure is composed of two "petals", and the $10 \mathrm{kV}$ line of each "petal" comes from the same busbar of the same substation and runs in a closed loop. The switch station section switches connected between the "double petals" operate in open loop, and each is standby, and the line utilization rate is $75 \%$. In addition, each switchyard is supported by two or more power points, and the load-to-power supply capacity is $100 \%$. The loss of any highvoltage substation will not lose load, but the scale of line construction is large, the switchyard is expensive, and the overall investment is high.

\section{3) Shanghai diamond type wiring}

The Shanghai Diamond Wiring is a dual-ring network structure with $10 \mathrm{kV}$ switch station as the core node, dualsided power supply, and self-healing function. It is composed of multiple substations, switch stations, and ring network rooms (boxes). It can realize 100\% load transfer between stations, and the reliability of power supply is high. In the case of $\mathrm{N}-1$, the $10 \mathrm{kV}$ line utilization rate is $50 \%$.

The structure is divided into the main network formed by the substation and switchyard, and the secondary backbone network formed by the switchyard and ring network room (box), as shown in Figure 5.

Due to the high cost of the switchyard, this wiring mode is more suitable for the transformation scheme of the distribution grid with the switchyard as the core node.

According to the characteristics of their own power grids and planning goals, cities in China and abroad have formed their own distinctive distribution network wiring modes according to local conditions, but they still have certain limitations in solving the current problems of the China's distribution network. Therefore, this article draws on the advantages of Tokyo three-radio wiring, 


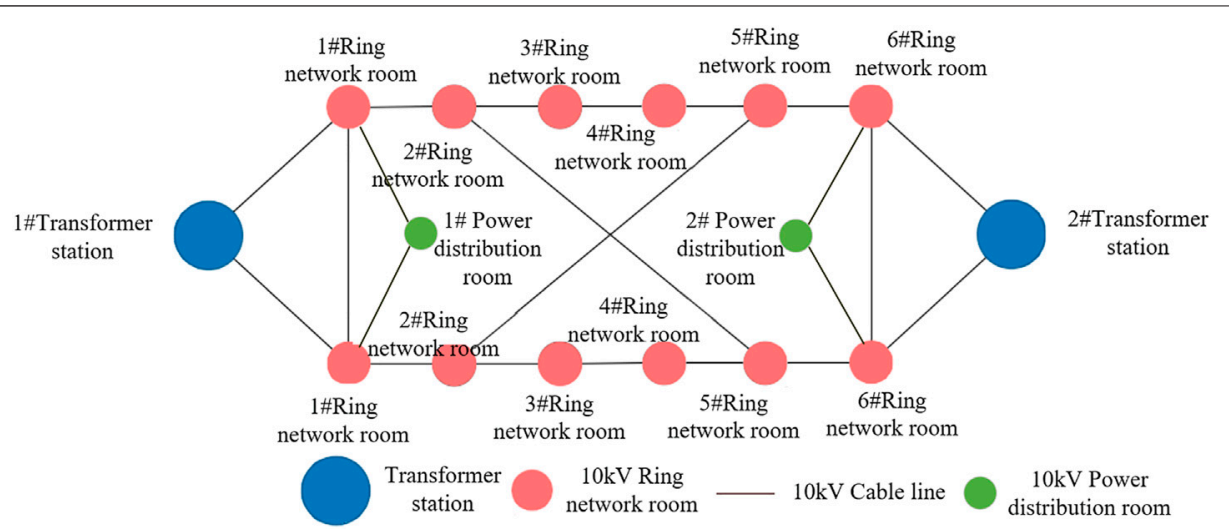

FIGURE 6 | Diamond-shaped distribution network structure.

Paris city three-ring network wiring, China's ring network room cable double-ring network wiring, "double petal" wiring, and Shanghai diamond wiring. Comprehensive consideration of adaptability, safety, flexibility, power supply reliability and economy, construct a diamond-shaped distribution network wiring mode with a clear structure.

\section{DIAMOND-SHAPED DISTRIBUTION NETWORK WIRING MODE}

In this paper, the $10 \mathrm{kV}$ diamond distribution network structure is based on the ring network room as the core node, using a hierarchical structure, divided into two levels of the main network and the bush network, with "all-circuit cable, full circuit breaker, all interconnection, full turn, all Self-healing "features, as shown in Figure 6.

The backbone network adopts different 110 (220) kV substations, four circuits of $10 \mathrm{kV}$ main lines fed from different sections of busbars and open loop operation. Each $10 \mathrm{kV}$ trunk line is equipped with three ring network rooms (boxes), and each $10 \mathrm{kV}$ trunk line is equipped with one contact in the station and two contacts between stations, forming a three-contact structure, and each ring network room (box) is equipped with three remotes. DTU and self-healing device. The branch network uses the power distribution room as an important user node, and uses different $10 \mathrm{kV}$ line ring network rooms (boxes) as the upper-level power source, and dual power sources are connected. The diamond-shaped distribution network structure plays a major role in improving the adaptability, safety, flexibility, power supply reliability, and economy of the distribution network.

\section{Adaptability, Security and Flexibility Analysis}

In the diamond-shaped distribution network connection, the incoming and outgoing lines of the ring network room are equipped with full circuit breakers and full protection. On the one hand, it can avoid the change of relay protection (Ma et al.,
2021) caused by the access of new elements such as distributed power sources and microgrids. On the other hand, it can solve the problem that the fuse curve of the fuse of the access point of largecapacity users is difficult to match with the zero-sequence current protection setting value, and meet the access needs of largecapacity users. Therefore, large-capacity users can be directly supplied by the substation to be connected to the ring network room for power supply, effectively reducing the direct supply of users from the substation. In addition, the improvement of line utilization efficiency will also help reduce the $10 \mathrm{kV}$ line outgoing line, and solve the problem of $10 \mathrm{kV}$ interval and outgoing channel tension in the substation.

The $10 \mathrm{kV}$ backbone network in the diamond-shaped distribution network connection supplies power to the twosided power supplies of different substations. The inter-station contact rate and inter-station load transfer capacity of the substation are both $100 \%$, which can ensure that the current level of power grids and the superior substation pass the N-1 check. Through the flexible adjustment of the operation mode, the planned power outage time during the overhaul period can be effectively reduced.

The diamond-type distribution network has a threeconnection structure, and the load transfer channels between stations are abundant. In the case of $\mathrm{N}-1$, the utilization rate of $10 \mathrm{kV}$ line equipment reaches $75 \%$, and the utilization rate of substations reaches $75 \%$ and above.

\section{Power Supply Reliability and Economic Analysis}

The diamond-type distribution network wiring is equipped with a self-healing system (Hu et al., 2021). The $10 \mathrm{kV}$ ring network room is equipped with a self-healing protection and control device according to the bus section, and each interval has a three-remote function to realize on-site information collection and fault self-healing.

The diamond-shaped distribution network wiring operates in an open loop, and when a single fault occurs, the line can be selfhealed and switched. The self-healing system uses optical fiber channel to exchange the switch and fault information between the 
TABLE 1 | Power supply reliability of different wiring modes.

\begin{tabular}{lc}
\hline Wiring mode & $\begin{array}{c}\text { Power supply } \\
\text { reliability } \\
\text { rate (\%) }\end{array}$ \\
\hline $\begin{array}{l}\text { Ring network room cable double ring network } \\
\text { connection }\end{array}$ & 99.99733 \\
$\begin{array}{l}\text { "Double petals" wiring } \\
\text { Thanghai Diamond Wiring }\end{array}$ & 99.99957 \\
\hline
\end{tabular}

ring network rooms, realizes the second-level recovery function of the non-faulty area in the case of a fault, and effectively guarantees the reliability of power supply and the ability of load transfer.

The failure mode consequence analysis method (Kebede et al., 2021; Zhang et al., 2021) is used to calculate the power supply reliability rate of each connection mode. Taking the diamondshaped distribution network structure in this article as an example, the power supply reliability rate (RS-1) calculation process is as follows:

Assuming that the loads are evenly distributed along the line and the probability of simultaneous failure or overhaul of two segments on a single feeder is 0 .
When the line fails, the user's power outage duration $T_{1}$ is calculated as:

$$
\begin{aligned}
T_{1}= & \frac{R}{\text { num }} \times \lambda \times \operatorname{num} \times t \times N \times\left(\frac{R \times \lambda}{\text { num }}\right)^{2} \times \frac{N}{2 \text { num }} \times\{[\text { num } \\
& \left.\left.\times \text { num }-\left(\sum_{i=1}^{\frac{\text { num }}{2}} i\right)\right] \times t+\text { num } \times\left(\frac{3 \text { num }}{4} \times t\right)\right\}
\end{aligned}
$$

where: $R$ is the length of the line, num is the number of segments, $N$ is the number of loads (users) carried by each outgoing line, $\lambda$ is the average failure rate of the line (times/kmyear), $t$ is the switching time of the line (hours/time).

When the switch fails, the user's power outage duration $T_{2}$ calculation formula is:

$$
T_{2}=\lambda \times 2 \times N \times\{(\text { num }-1) \times t\}
$$

When the distribution transformer fails, the user's power outage duration $T_{3}$ calculation formula is:

$$
T_{3}=N \times \lambda \times\{r+(\text { num }-1) \times t\}
$$

where: $r$ is the average repair time of the line (hours/time).

The user's total power outage duration $T$ is calculated as:

$$
T=T_{1}+T_{2}+T_{3}
$$

TABLE 2 | Economic analysis of newly-built typical power supply grid.

\section{Wiring mode}

\section{Switch station/ring}

network

room (ten thousand

dollars)

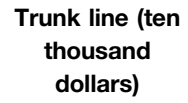

Trunk line (ten thousand dollars)

83.20

246.53

369.80

110.94

\section{Protection/distribution \\ automation \\ (ten thousand dollars)}

Total

(ten thousand

dollars)

$\begin{array}{ccc}244.07 & 110.94 & 438.21 \\ & & \\ 273.65 & 55.47 & 575.65 \\ 277.35 & 97.07 & 744.22 \\ 207.09 & 107.86 & 425.89\end{array}$

The diamond-shaped distribution network structure

Note: The unit price of the switch station is 30.82 ten thousand dollars/seat, the unit price of the ring room is 4.62 ten thousand dollars/seat, the cable line unit price of 18.49 ten thousand dollars $/ \mathrm{km}$, distribution automation device 3.08 ten thousand dollars/set, and the protection and self-healing system 7.7 ten thousand dollars/set.
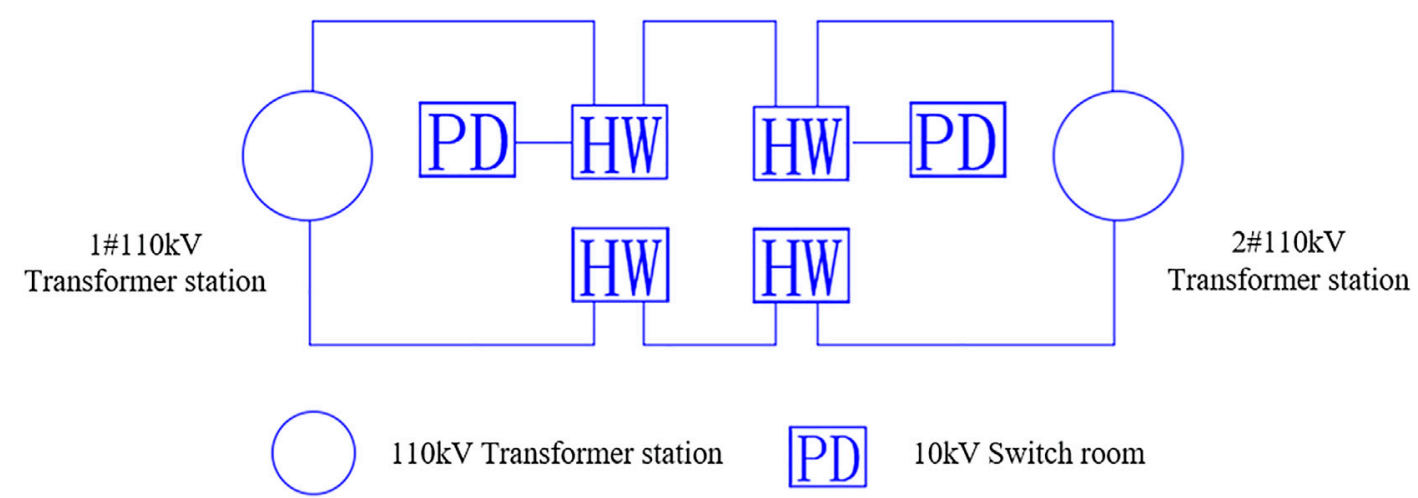

$110 \mathrm{kV}$ Transformer station

PD 10kV Switch room

$\mathrm{HW}$ Existing $10 \mathrm{kV}$ ring network room

Current $10 \mathrm{kV}$ ring network room

FIGURE 7 | Schematic diagram of the current distribution network wiring mode in the demonstration area. 


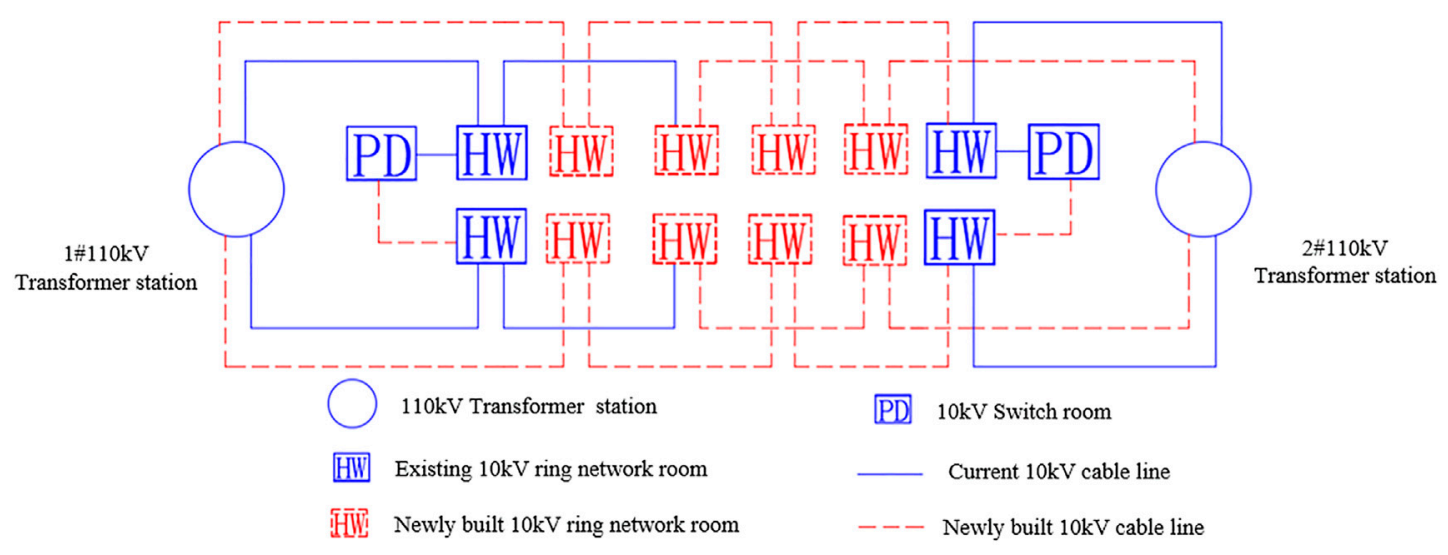

FIGURE 8 | The first wiring mode modification plan.

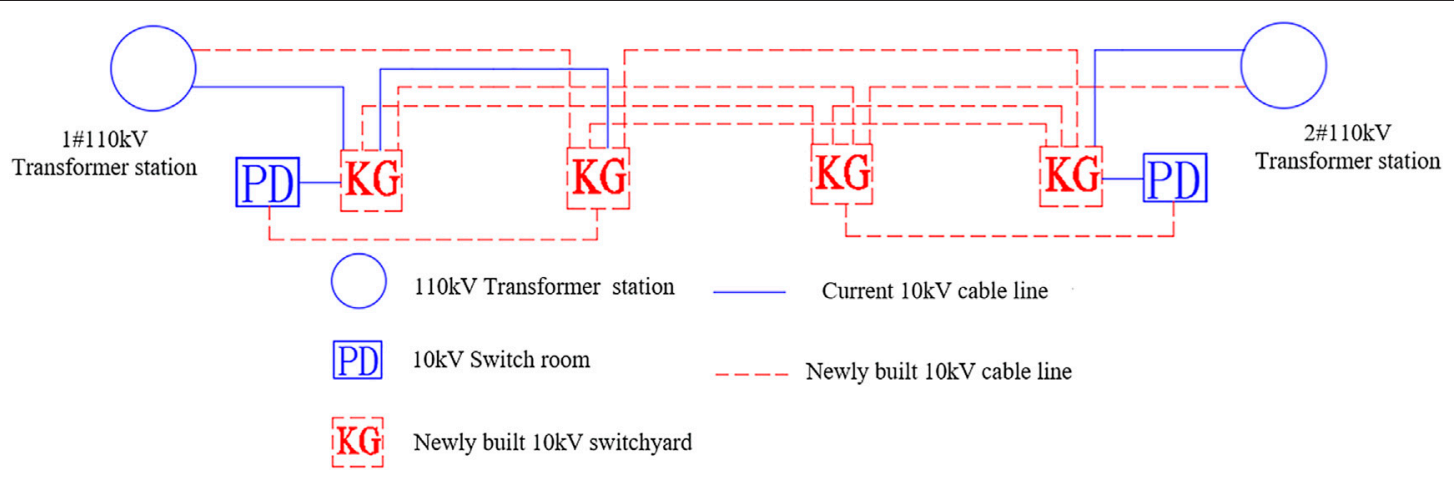

FIGURE 9 | The second wiring mode modification plan.

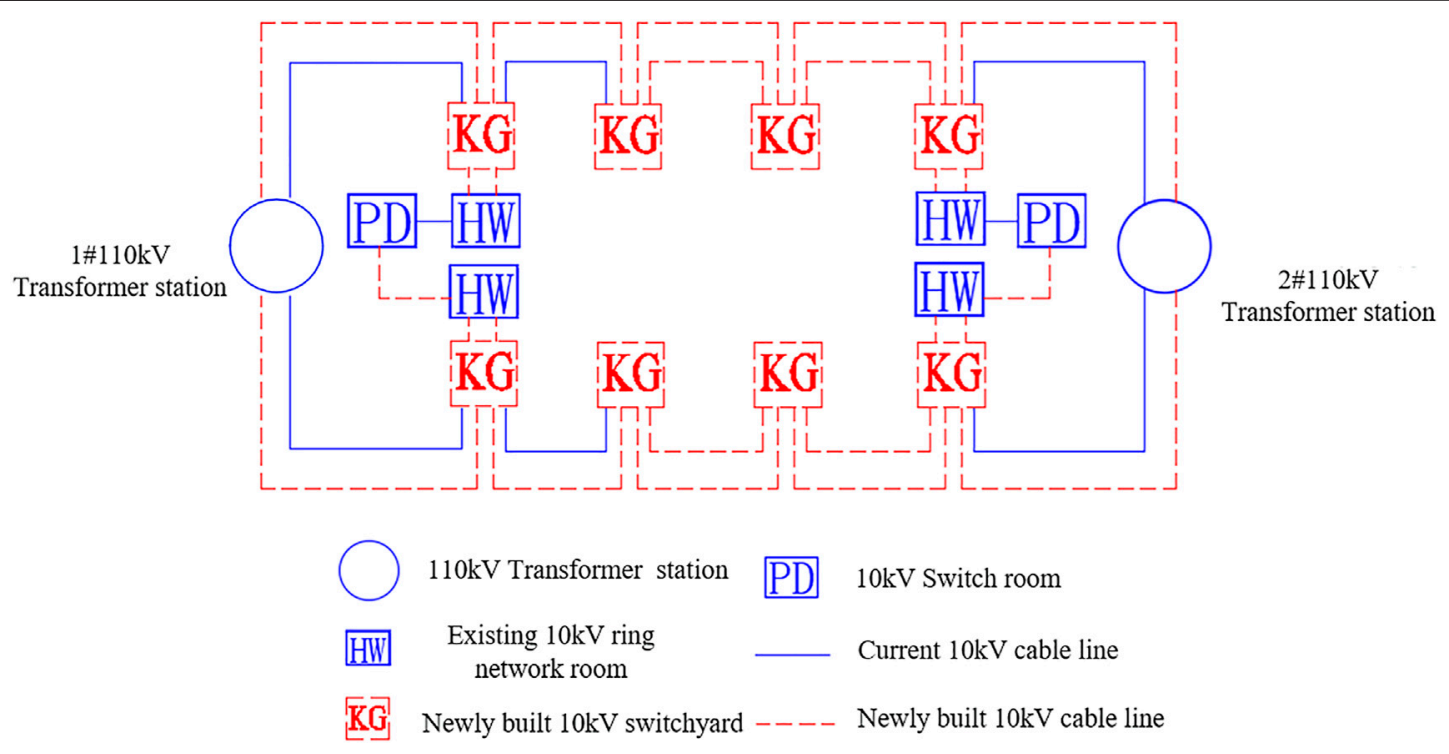

FIGURE 10 | The third wiring mode modification plan. 

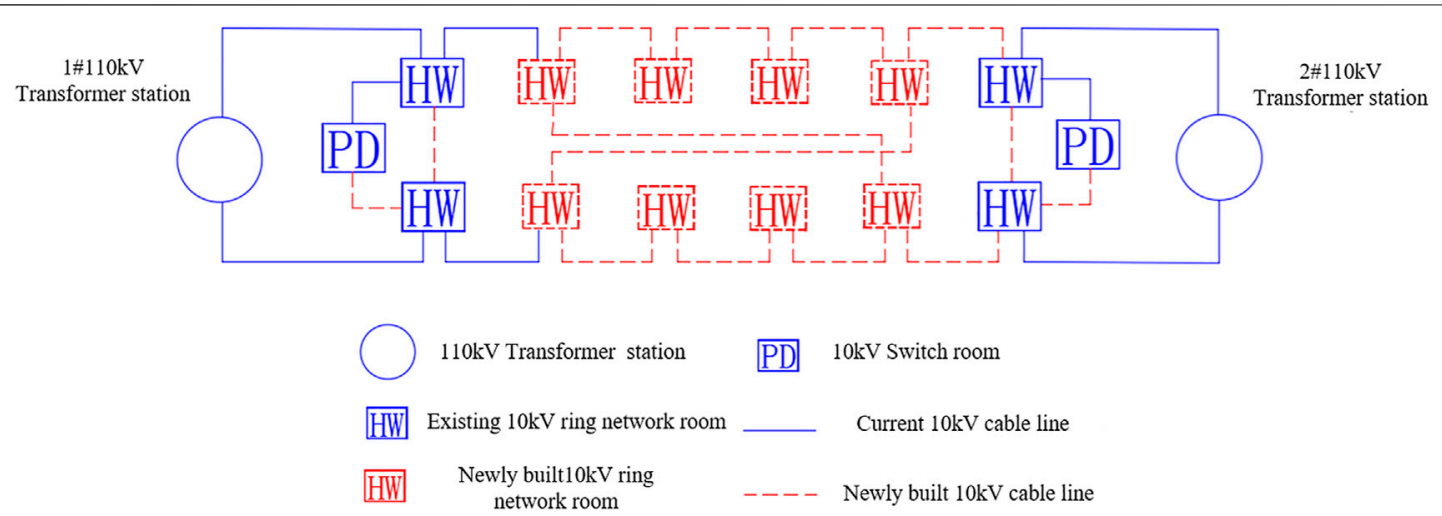

FIGURE 11 | The fourth wiring mode modification plan.

TABLE 3 | The effect of the four wiring mode transformation schemes.

Wiring mode

\begin{tabular}{cccc}
\multicolumn{2}{c}{ Index } \\
\hline Adaptability & Security and flexibility & $\begin{array}{c}\text { Power supply } \\
\text { reliability }\end{array}$ & Economy
\end{tabular}

Ring network room cable double ring network connection

"Double petals" wiring

Shanghai Diamond Wiring

The diamond-shaped distribution network structure in this paper
Occupies $810 \mathrm{kV}$ intervals

Occupies $410 \mathrm{kV}$ intervals

Occupies $810 \mathrm{kV}$ intervals

Occupies $410 \mathrm{kV}$ intervals

\section{Meet the $\mathrm{N}-1$ calibration under the condition of overhaul,} and the line utilization efficiency is still $50 \%$

Meets the N-1 calibration under the condition of overhaul, and the line utilization efficiency is increased to $75 \%$

Meet the N-1 calibration under the condition of overhaul, and the line utilization efficiency is still $50 \%$

Meets the $\mathrm{N}-1$ calibration under the condition of overhaul, and the line utilization efficiency is increased to $75 \%$
Increase $0.00472 \%$

Increase $0.00696 \%$ Increase $0.007 \%$

Increase $0.00704 \%$
Investment of 147.92 ten thousand dollars Investment of 256.39 ten thousand dollars

Investment of 357.47 ten thousand dollars Investment of 110.94 ten thousand dollars
The power supply reliability rate $(R S-1)$ calculation formula is:

$$
R S-1=1-\frac{T}{N \times 8760}
$$

According to the above formula, the power supply reliability rate of the diamond-shaped distribution network in this article is $99.99965 \%$. In the same way, the power supply reliability of other wiring modes can be calculated, and the calculation results are shown in Table $\mathbf{1 .}$

When the boundary conditions are the same, the power supply reliability rate of the diamond-type wiring in this paper is about $0.00232 \%$ higher than that of the double-loop network wiring of the ring network room cable, about $0.00008 \%$ higher than the "double petal" wiring, and about $0.00004 \%$ higher than the Shanghai diamond-type wiring.

In the same power supply grid, respectively construct ring network room cable double ring network wiring, "double petal" wiring, Shanghai diamond wiring and this article diamond wiring. Taking the grid area of the power grid of $2 \mathrm{~km}^{2} \mathrm{n} 2$ and the saturated load of $70 \mathrm{MW}$ as an example, the investment scale of each new typical grid of the power grid is calculated.

The calculation formula for the investment scale of each connection mode is:

$$
S=S_{1}+S_{2}+S_{3}
$$

where: $S_{1}$ is investment in switchyard or ring network room, $S_{2}$ is investment in backbone line, $S_{3}$ is investment in protection or distribution automation.

According to the above formula, the economic situation of each wiring mode is shown in Table 2.

The investment in the diamond-type distribution network proposed in this paper is about 3\% lower than the investment in the double-ring network of the ring room cable, about $26 \%$ lower than the "double petal" investment, and about $43 \%$ lower than the Shanghai diamond-type investment, which has higher economic efficiency.

\section{EXAMPLE APPLICATION}

\section{Empirical Regional Current Distribution Network Wiring Mode}

Taking a $10 \mathrm{kV}$ distribution network in a certain area of a city as an example, the power supply reliability of the grid in this area is $99.99261 \%$, the current load is $22 \mathrm{MW}$, and it is powered by two sets of cable single loop networks, and the power supply is $110 \mathrm{kV1 \#}$, two\# substations, as shown in Figure 7.

Before the transformation, the regional grid did not meet the "N-1" check under the maintenance mode, the power distribution room was powered by a single power source, and 
the utilization efficiency of the $10 \mathrm{kV}$ line was $50 \%$. The load rate of $1 \#$ and 2\# substations is seriously unbalanced. The load rate of 1 \# substation is $81.9 \%$, the interval utilization rate is $91.67 \%$, and the remaining intervals are few. The load rate of $2 \#$ substation is only $25.32 \%$, and the interval utilization rate is $54.17 \%$.

\section{Connection Mode Modification Plan}

The result of the load forecast for this region in 2025 is $35 \mathrm{MW}$. The transformation schemes for the four wiring modes are shown below.

Option 1: Transform the cable single-ring network connection of the demonstration area into the double-ring network cable connection of the ring network room, and it is necessary to construct two sets of target connections, as shown in Figure 8.

Option 2: Transform the single-ring network connection of the cable in the demonstration area ring network room into a "double petal" connection, and a set of target connections needs to be constructed, as shown in Figure 9.

Option 3: Transform the cable single ring network connection of the demonstration area ring network room into a Shanghai diamond type connection, and two sets of backbone connections need to be constructed, as shown in Figure 10.

Option 4: Transform the single-ring network connection of the cable in the demonstration area ring network room into the diamond-type connection in this article, and a set of target connections needs to be constructed, as shown in Figure 11.

Table 3 shows the effect of the four wiring mode transformation schemes.

Adaptability: the cable double loop network and Shanghai diamond wiring mode occupy eight intervals, and the cable "double petals" and diamond wiring patterns occupy only four intervals, which effectively alleviates the $10 \mathrm{kV}$ interval and the tension of the outlet channel in the substation.

Security and flexibility: After the transformation of the four schemes, the $2 \# 110 \mathrm{kV}$ substation can be transferred to the $1 \# 110 \mathrm{kV}$ substation part load, and the power distribution room is powered by dual power supplies, and all meet the N-1 check under the condition of maintenance. But the utilization efficiency of the cable double-ring network and the Shanghai diamond-shaped wiring mode $10 \mathrm{kV}$ line are still $50 \%$, and the cable "double petals" and the diamond-shaped wiring mode $10 \mathrm{kV}$ line utilization efficiency has increased to $75 \%$.

Power reliability: the increase in power supply reliability from high to low is the diamond-type wiring in this paper, Shanghai diamond-type wiring, cable "double petals", and cable doublering network wiring. The increase values are 0.00704, 0.007, 0.00696 , and $0.00472 \%$ respectively.

Economy: The scale of investment from small to large is the diamond-shaped wiring in this paper, the double-loop cable network, the cable "double petals", and the Shanghai diamond-shaped wiring. Among them: the utilization rate of the original cable line in the diamond connection mode in this paper is about $100 \%$, with a total investment of $1,109,400$ dollars; the utilization rate of the original cable line in the cable double-ring network connection mode is about $100 \%$, with a total investment of $1,479,200$ dollars. It is about $33.33 \%$ higher than the diamond type wiring; The cable "double petals" connection mode transforms the original $10 \mathrm{kV}$ ring network room into a switch station. The total investment is 2.5639 million dollars, which is about $131.1 \%$ higher than the diamond connection mode; the original cable line utilization rate of the Shanghai diamond connection mode is about $100 \%$. It need to build $810 \mathrm{kV}$ switch stations as the core nodes of the distribution network structure, with a total investment of 3.5747 million dollars, which is about $222.22 \%$ higher than the diamond wiring.

Overall consideration of adaptability, safety, flexibility, power supply reliability, and improvement of economic indicators, the diamond-type distribution network wiring has good economy and operability.

\section{CONCLUSION}

This paper studies a diamond-shaped distribution network structure with a clear structure. The advantages of this structure are: 1) It has strong adaptability and can solve the problem of tight $10 \mathrm{kV}$ intervals in substations; 2) It has high power supply security and flexibility, which can meet the N-1 check under the maintenance mode, the load transfer rate is $100 \%$, the $10 \mathrm{kV}$ line utilization rate can reach $75 \%$, and the substation utilization rate can reach $75 \%$ and above; 3 ) The entire line is equipped with a self-healing system, which can realize the second-level recovery function in the non-faulty area in the event of a fault, and the power supply reliability is high; 4) In areas where the ring network room is the core node of the distribution network frame, the distribution network can be reconstructed on the basis of the original ring network room and incoming and outgoing lines, which is economical.

The research results have been applied to the structural transformation of the distribution network in a certain area of a certain city, and verified the feasibility and superiority of the diamond-shaped distribution network structure.

\section{DATA AVAILABILITY STATEMENT}

The original contributions presented in the study are included in the article/Supplementary Material, further inquiries can be directed to the corresponding author.

\section{AUTHOR CONTRIBUTIONS}

LS was responsible for the specific work of this paper. WJ and XL guided the work of this article. WD and LH carried out some of the calculation work. LW conducted data collection, WS and LW carried out the index calculation and comparison of the scheme.

\section{FUNDING}

The authors acknowledge the funding of the State Grid Corporation of China's Science and Technology Project (JSB17202000260). 


\section{ACKNOWLEDGMENTS}

The corresponding author thanks State Grid Zhejiang Pinghu Power Supply Co., Ltd. for its selfless support and Tianjin Tianda Qiushi New Power Technology Co., Ld. for its support in the design of the scheme.

\section{REFERENCES}

Chen, S., and Liu, G. (2020). Fault Recovery Method for Distribution Network with Distributed Power Access. Electr. Meas. Instrumentation. 57 (18), 50-56. doi:10.19753/j.issn1001-1390.2020.18.009

Feng, J., Chen, J. Y., and Chen, M. J. (2015). Research on Graph Theory-Based Service Restoration Algorithm of Distribution Network Containing Distributed Generation. Electric Power. 48 (6), 63-71.

Hu, H., Yu, S. S., Zhao, J., Chau, T., Ding, F., Fernando, T., et al. (2021). MPC-based Double-Layer Real-Time Conditional cSelf-Restoration for Interconnected Microgrids. Int. J. Electr. Power Energ. Syst. 129, 106745. doi:10.1016/ j.ijepes.2020.106745

Hu, X. F., and Li, Y. Y. (2017). Optimization Method of Substation bay Resources Based on Hierarchical Model. China Electric Power. 50 (7), 175-179. doi:10.11930/j.issn.1004-9649.2017.07.175.05

Kebede, F.-S., Olivier, J.-C., Bourguet, S., and Machmoum, M. (2021). Reliability Evaluation of Renewable Power Systems through Distribution Network Power Outage Modelling. Energies 14 (11), 3225. doi:10.3390/en14113225

Li, P., Mi, Y., Liu, N., Zhou, Y., Yu, H., and Ji, H. (2021). Editorial: Flexible and Active Distribution Networks. Front. Energ. Res. 9. doi:10.3389/fenrg.2021.687129

Liang, C., Tang, C. H., Song, H. X., and Feng, X. X. (2018). Analysis of the Practice and Typical Characteristics of World-Class Power Distribution Network. DEStech Trans. Comp. Sci. Eng. 332, 539-542. doi:10.12783/dtcse/ccme2018/ 28664

Lin, R. T., and Lin, Z. H. (2020). Power User Outage Impact index System and Comprehensive Evaluation Method. Power Syst. Tech. 58 (6), 2350-2360. doi:10.13335/j.1000-3673.pst.2019.1146

Liu, X. N., Wei, J., and Zhang, W. T. (2019). Investment Benefits Evaluation and Decision for Distribution Network Based on Information Entropy and Fuzzy Analysis Method. Power Syst. Prot. Control. 47 (12), 48-56. doi:10.19783/ j.cnki.pspc. 180965

Liu, Y., Zhang, L., Xi, L., Sun, Q., and Zhu, J. (2021). Automatic Generation Control for Distributed Multi-Region Interconnected Power System with Function Approximation. Front. Energ. Res. 9, 1-12. doi:10.3389/fenrg.2021.700069

Ma, G. Z., Jiang, L., Shao, H., Wang, K., Ling, Y. P., Ling, Y. P., et al. (2015). Optimal Combination of Load and its Application on Urban Power Network Renovation. 2015 2nd Asian Pacific Conference on Energy, Environment and Sustainable Development (APEESD 2015) Paris, France.

Ma, K., Chen, Z., Liu, Z., Leth Bak, C., and Castillo, M. (2021). Protection Collaborative Fault Control for Power Electronic-Based Power Plants during Unbalanced Grid Faults. Int. J. Electr. Power Energ. Syst. 130, 107009. doi:10.1016/j.ijepes.2021.107009

Narayanan, V., Kewat, S., and Singh, B. (2021). Implementation of a Multiobjective Control for Islanded Hybrid Microgrid. IEEE Trans. Ind. Applicat. 57 (3), 2702-2713. doi:10.1109/TIA.2021.3066327

Pragathi, B., Poonia, R. C., Polaiah, B., and Nayak, D. K. (2021). Evaluation and Analysis of Soft Computing Techniques for Grid Connected Photo Voltaic System to Enhance Power Quality Issues. J. Electr. Eng. Technol. 16, 1833-1840. doi:10.1007/s42835-021-00720-w

Shang, Y., Shi, S., and Dong, X. (2015). Islanding Detection Based on Asymmetric Tripping of Feeder Circuit Breaker in Ungrounded Power Distribution System. J. Mod. Power Syst. Clean. Energ. 3 (4), 526-532. doi:10.1007/s40565-0150162-7

\section{SUPPLEMENTARY MATERIAL}

The Supplementary Material for this article can be found online at: https://www.frontiersin.org/articles/10.3389/fenrg.2021.735294/ full\#supplementary-material

Song, R. C., Yang, Q., and Tang, Y. J. (2015). Comparative Study on HighReliability Connection Modes for Switching Station in Distribution Network. Power Syst. Tech. 39 (3), 769-775. doi:10.13335/j.1000-3673.pst.2015.03.028

Wang, Y., and Jiang, S. (2020). Research on the Construction of Urban MediumVoltage Distribution Network Grids that Meet the Demand for High-Reliability Power Supply. Power Supply 6, 33-37. doi:10.19421/j.cnki.10066357.2020.06.006

Xiao, J., and She, B. X. (2019). Comprehensive Efficiency Evaluation of Distribution Network Connection Mode. Power Syst. Tech. 43 (10), 3769-3781. doi:10.13335/j.1000-3673.pst.2018.2973

Xu, B. Y. (2017). - Basic Components. Power Supply 4 (2), 14-67. doi:10.1201/ b15015-4

Zhang, K., and Troitzsch, S. (2021). Robust Scheduling for Networked Microgrids under Uncertainty. Front. Energ. Res. 9, 1-17. doi:10.3389/fenrg.2021.632852

Zhang, K., Zhou, B., Or, S. W., Li, C., Chung, C. Y., and Voropai, N. I. (2021). Optimal Coordinated Control of Multi-Renewable-To-Hydrogen Production System for Hydrogen Fueling Stations. IEEE Trans. Ind. Applicat. 57, 1-11. doi:10.1109/TIA.2021.3093841

Zhang, L. D., and Dou, X. (2018). Research on Distribution Network Planning under the Background of Integrated Energy. Power Supply. 35 (4), 37-45. doi:10.19421/j.cnki.1006-6357.2018.04.007

Zhao, F. X., and Wu, J. (2016). Intelligent Distribution Network Based on MAPSO Optimization of Large-Scale Outage Power Supply Restoration. China Electric Power. 49 (1), 85-90. doi:10.11930/j.issn.1004-9649.2016.01.085.06

Zhou, B., Zhang, K., Chan, K. W., Li, C., Lu, X., Bu, S., et al. (2021a). Optimal Coordination of Electric Vehicles for Virtual Power Plants with Dynamic Communication Spectrum Allocation. IEEE Trans. Ind. Inf. 17 (1), 450-462. doi:10.1109/tii.2020.2986883

Zhou, X., Chang, C.-Y., Bernstein, A., Zhao, C., and Chen, L. (2021b). Economic Dispatch with Distributed Energy Resources: Co-optimization of Transmission and Distribution Systems. IEEE Control. Syst. Lett. 5 (6), 1994-1999. doi:10.1109/LCSYS.2020.3044542

Conflict of Interest: LS, WJ, XL, and WD was employed by State Grid Zhejiang Pinghu City Power Supply Co., Ltd. LWl, WS, and LWq was employed by Tianjin Tianda Qiushi New Power Technology Co., Ld

The remaining author declares that the research was conducted in the absence of any commercial or financial relationships that could be construed as a potential conflict of interest.

Publisher's Note: All claims expressed in this article are solely those of the authors and do not necessarily represent those of their affiliated organizations, or those of the publisher, the editors, and the reviewers. Any product that may be evaluated in this article, or claim that may be made by its manufacturer, is not guaranteed or endorsed by the publisher.

Copyright (c) 2021 Shengli, Jun, Longjiang, Di, Haiqing, Wenlong, Shaofang and Wenqi. This is an open-access article distributed under the terms of the Creative Commons Attribution License (CC BY). The use, distribution or reproduction in other forums is permitted, provided the original author(s) and the copyright owner(s) are credited and that the original publication in this journal is cited, in accordance with accepted academic practice. No use, distribution or reproduction is permitted which does not comply with these terms. 